\title{
Mineração
}

\section{Redução da variabilidade da qualidade do carvão com otimização do tamanho de pilhas de homogeneização}

\author{
Reducing coal quality variability with size \\ optimization of blended piles
}

\author{
Filipe Schmitz Beretta \\ Engenheiro de Minas \\ Estudante de Mestrado, UFRGS \\ fberetta@ymail.com \\ João Felipe Coimbra Leite Costa \\ Engenheiro de Minas \\ Prof. PhD. DEMIN, UFRGS \\ jfelipe@ufrgs.br
}

Jair Carlos Koppe

Engenheiro de Minas

Prof. Titular, Dr., DEMIN, UFRGS

jkoppe@ufrgs.br

\section{Resumo}

A variabilidade do minério in-situ é um dos grandes problemas em todas as etapas pós-lavra, pois comportamentos erráticos de parâmetros físico-químicos podem acarretar grandes dificuldades para o processamento do minério. Pilhas de estoque tem sido utilizadas para reduzir a flutuabilidade de teores, servindo como um filtro da variabilidade. O principal parâmetro para a redução da variabilidade na pilha é a massa de minério estocado. Quanto maior a pilha, menor a variabilidade. Porém há limites viáveis para sua construção. $\mathrm{O}$ incorreto dimensionamento leva a perdas financeiras, devido aos altos custos de estoque e às perdas em recuperação e teores na usina de beneficiamento. Esse estudo propõe quantificar a redução da variabilidade de conteúdo de cinza e teor de enxofre em um depósito de carvão. A estimativa de teores em modelos de blocos por métodos tradicionais como krigagem ordinária não considera as incertezas associadas ao modelo geológico. Dessa forma, a utilização de simulação geoestatística é mais adequada para prever flutuações locais dos teores. Para as variáveis testadas, nesse estudo, foram analisadas as reduções de flutuação por meio de diferentes tamanhos de pilhas.

Palavras-chave: Homogeneização, pilhas, simulação geoestatística.

\section{Abstract}

The in-situ grade variability imposes a series of problems during ore processing. Blending is commonly used to reduce this variability. The main factor to reduce head grade variability is the pile mass and size. The larger the pile, the lesser the reclaimed grades vary. However, there are practical limits to the pile length and mass. An incorrect design leads to financial losses due to either high capital costs for oversized piles or losses in plant recovery due to undersized piles incapable of attenuating grade fluctuations. Block estimation by traditional methods, such as ordinary krigging, does not properly incorporate the uncertainty associated with the estimates. Therefore, geostatistical simulation was selected. as it is the best suited to predict local grade fluctuations. The variables used to illustrate the proposed methodology were ash and sulfur content, for which the reduction of the fluctuation was analyzed for different pile sizes.

Keywords: Blending, piles, geostatistical simulation. 


\section{Introdução}

O desenvolvimento econômico e social de uma região depende, entre outros fatores, diretamente da energia disponível para processos industriais e consumo residencial. Essa demanda energética acarreta, muitas vezes, na ampliação da matriz energética. Em alguns locais, centrais termelétricas alimentadas a carvão são ampliadas ou instaladas. Algumas dessas termoelétricas são projetadas para receber o produto bruto lavrado ou run of mine (ROM), o que permite a maximização da recuperação, redução de custos operacionais e de impactos ambientais provenientes de plantas de beneficiamento. Porém algumas variáveis físico-químicas do carvão devem ser mantidas dentro de intervalos aceitáveis para o bom funcionamento da termoelética e a utilização direta do combustível (carvão ROM) faz com que a sua variação intrínseca de qualidade afete, constantemente, a eficiência do processo de combustão.

Pilhas de homogeneização são frequentemente, utilizadas para filtrar essa variabilidade, além de garantir certo

\section{Metodologia}

Para que a alimentação das usinas termoelétricas consumidoras de carvão seja feita conforme os padrões necessários e garanta a viabilidade do projeto, as pilhas devem ser formadas com o objetivo de filtrar a variabilidade dos atributos do carvão in situ em cada bloco simulado.

As variáveis utilizadas, para ilustrar a redução da flutuabilidade da qualidade, estoque de minério. Os principais parâmetros de construção da pilha são o tipo de disposição, número de camadas e massa incorporada. Este último é o principal redutor da variabilidade dos teores, tendo em vista que, quanto maior a massa homogeneizada, menor é a variabilidade entre os lotes (David, 1977).

A sequência do material depositado na pilha, também, influencia na previsão do teor médio de cada pilha, o que exige uma previsão de avanço de lavra. Esse sequenciamento traduz os valores da reserva de minério (modelo 3D) em um vetor de teores lavrados ao decorrer do tempo de atividade (modelo 1D). Para tanto, é indispensável a utilização de técnicas geoestatísticas para prever os teores que compõe os blocos a serem lavrados. A técnica de krigagem é um ótimo estimador de teores, porém não é adequada para se preverem incerteza associadas às estimativas (Isaaks \& Srivastava, 1989). Em função dessa restrição, faz-se necessária a utilização de técnicas de simulações condicionais, que possibilitam um melhor modelamento da incerteza pois combina as várias realizações. O modelo é, condicionalmente, simulado, se reproduzir as mesmas características de dispersão do conjunto de dados original, tal como a média, variância e, covariância (Costa et al., 2008).

Nesse estudo, será verificada a influência do volume homogeneizado na redução da flutuabilidade da qualidade do carvão entre vários lotes (ou pilhas) produzidos para atender a demanda de uma unidade termoelétrica. O estudo de caso é feito em um depósito de carvão no sul do Brasil e verifica qual o tamanho de lote que seja capaz de atenuar a variabilidade, a ponto de produzir lotes com baixa probabilidade de exceder o conteúdo de cinza médios em $58,5 \%$ e teores de enxofre em $2,2 \%$. Para isto, foi necessário o desenvolvimento de uma metodologia baseada na construção de um modelo de blocos validado por simulação geoestatística, respeitando uma sequência de lavra preestabelecida, na qual foram quantificadas as flutuações dos atributos de interesse para vários tamanhos de pilhas (ou lotes). foram o conteúdo de cinzas e o teor de enxofre. As variáveis foram simuladas para cada bloco de $50 \mathrm{~m} \times 50 \mathrm{~m}$, para seis camadas de carvão, utilizando simulação por método de bandas rotativas (Journel, 1974), que permite acessar a incerteza associada à previsão dos valores antes mesmo de tentar alguma predição sobre os valores da própria variável (Gambin et al., 2005). Com isto, foi possível gerar vários cenários equiprováveis de distribuição dos teores.

Os modelos gerados, nas simulações, foram convertidos em pilhas de diversos tamanhos, com seus teores baseados no sequenciamento determinado a partir de informações operacionais. A seqüência de extração foi estabelecida

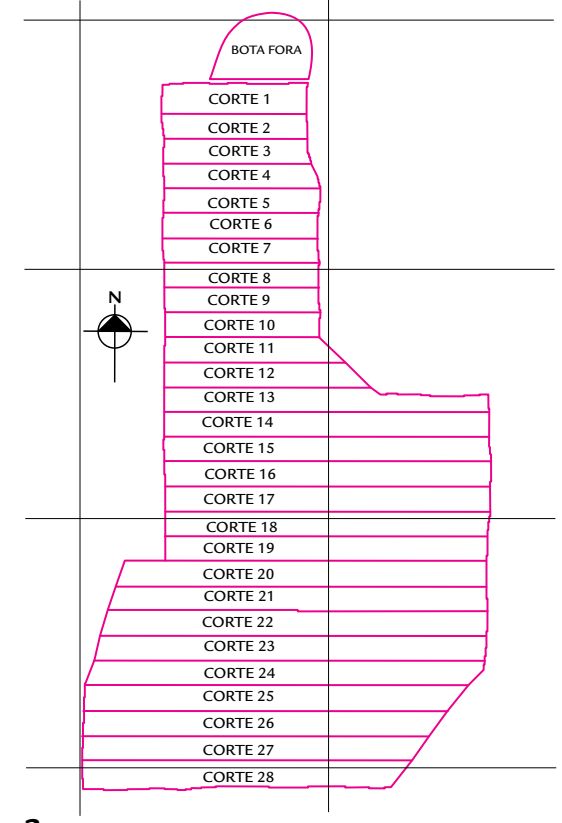

a

\begin{tabular}{|c|c|c|c|c|}
\hline 5 & 6 & 15 & 16 & 25 \\
\hline 4 & 7 & 14 & 17 & 24 \\
\hline 3 & 8 & 13 & 18 & 23 \\
\hline 2 & 9 & 12 & 19 & 22 \\
\hline 1 & 10 & 11 & 20 & 21 \\
\hline
\end{tabular}

b
Figura 1

Área destinada à lavra, dividida em 28 cortes (a), detalhe de um corte onde os blocos foram extraídos na sequência estabelecida (b). 
em cortes representando a produção média anual. A lavra inicia a sudoeste do corte 1 , migrando para norte até o fim do corte, retornando ao sul no próximo bloco (Figura 1). Ao término de cada corte, as operações retornam de leste para oeste, para que não haja deslocamento dos equipamentos de lavra. Os blocos de camadas que sofreram erosão foram desconsiderados e a lavra segue a partir do bloco subsequente. A seqüência de cortes avança de norte para sul.

Portanto, para se garantir que os objetivos citados fossem atingidos, a seguinte metodologia foi proposta nesse trabalho:

I. Revisão, preparação e análise do banco de dados.

II. Construção de modelos equiprováveis de distribuição de conteúdos de cinzas e teores de enxofre.

III.Sequenciamento da extração dos

\section{Resultados e discussão}

As amostras foram divididas em seis domínios referentes às camadas. Os dados disponíveis não estão em mesmo número entre os atributos, nem entre as camadas. O conteúdo de cinzas e o teor de enxofre foram amostrados em alguns poucos furos de sondagem. As espessuras, por serem amostras mais simples de serem obtidas, estão em número maior (mais de

\section{0 amostras em todas as camadas).}

Para que o comportamento da variância dos dados pudesse ser estimado de forma mais confiável, a variografia foi feita com base nos dados de espessura, pois a baixa quantidade de amostras dos parâmetros de qualidade impede que bons variogramas pudessem ser feitos em algumas das camadas.
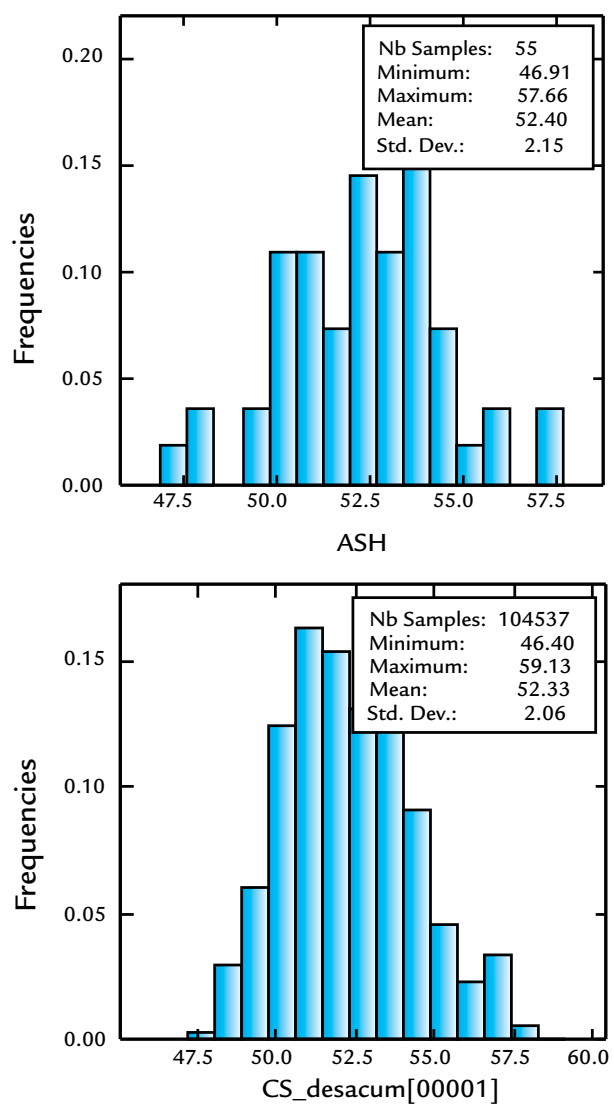

Histograma dos dados amostrais de cinza nas camadas Superior (a) e Inferior (b) e os respectivos histogramas dos resultados simulados (c) e (d) para conteúdos de cinza, em uma simulação aleatoriamente escolhida. do em variáveis acumuladas de unidade $\%$.m. Dessa forma, todas as amostras passam a pertencer ao mesmo suporte, quando trabalhadas na forma de variável auxiliar (acumulação).

Para que o resultado final possa ser expressado em porcentagem de cinza e enxofre, é necessário simular, também, a espessura, que servirá como divisor em cada bloco na retrotransformação da variável auxiliar acumulação para a variável de interesse. Em cada bloco a ser extraído, é incorporada a massa e, também, são incorporados os teores das seis camadas, que juntas compõem o valor final do atributo no bloco ponderado pela contribuição mássica de cada camada com seus respectivos teores de enxofre e cinza. Assim, foram previstos os impactos na redução da variabilidade de teor de enxofre e conteúdo de cinzas nos lotes compostos pelos blocos.

As estratégias e os parâmetros de busca foram definidos iterativamente, de forma que todos os blocos pudessem ser simulados. Garantiu-se, assim, que toda a área reservada para a lavra fosse contemplada pela simulação, mesmo que, para isso, a confiabilidade dos valores simulados, para locais com menos amostras, fosse alta (fator falta de informação).
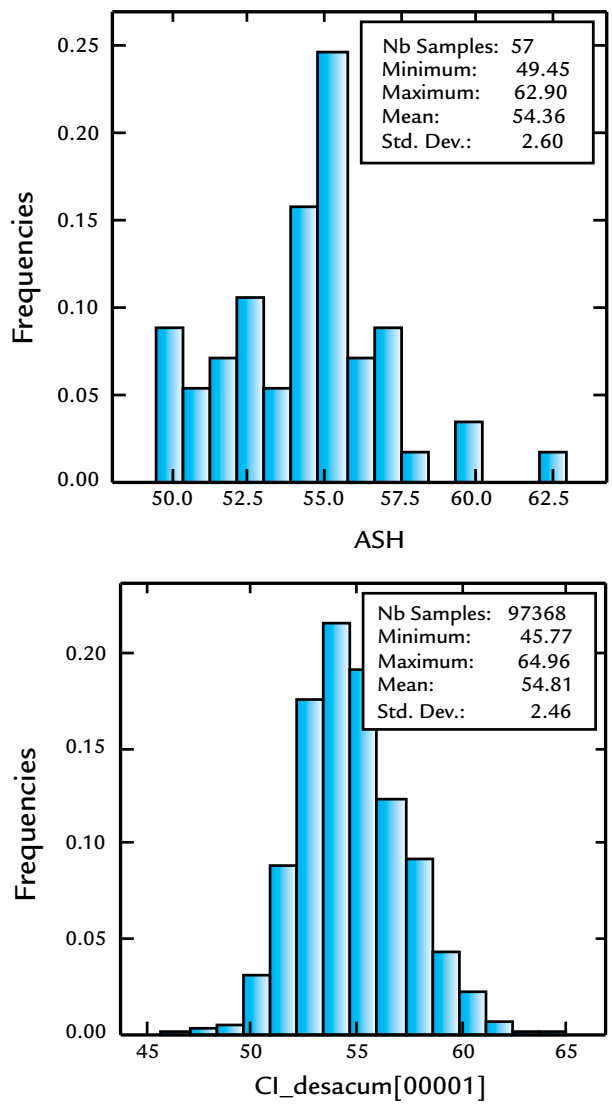
Foram realizadas 40 simulações, cada uma retornando com um valor equiprovável para cada bloco simulado. Dessa forma, os valores simulados levaram a diferentes teores de lotes, mesmo seguindo a mesma combinação da sequência de lavra.

A validação das simulações foi feita pela comparação entre os dados condi-
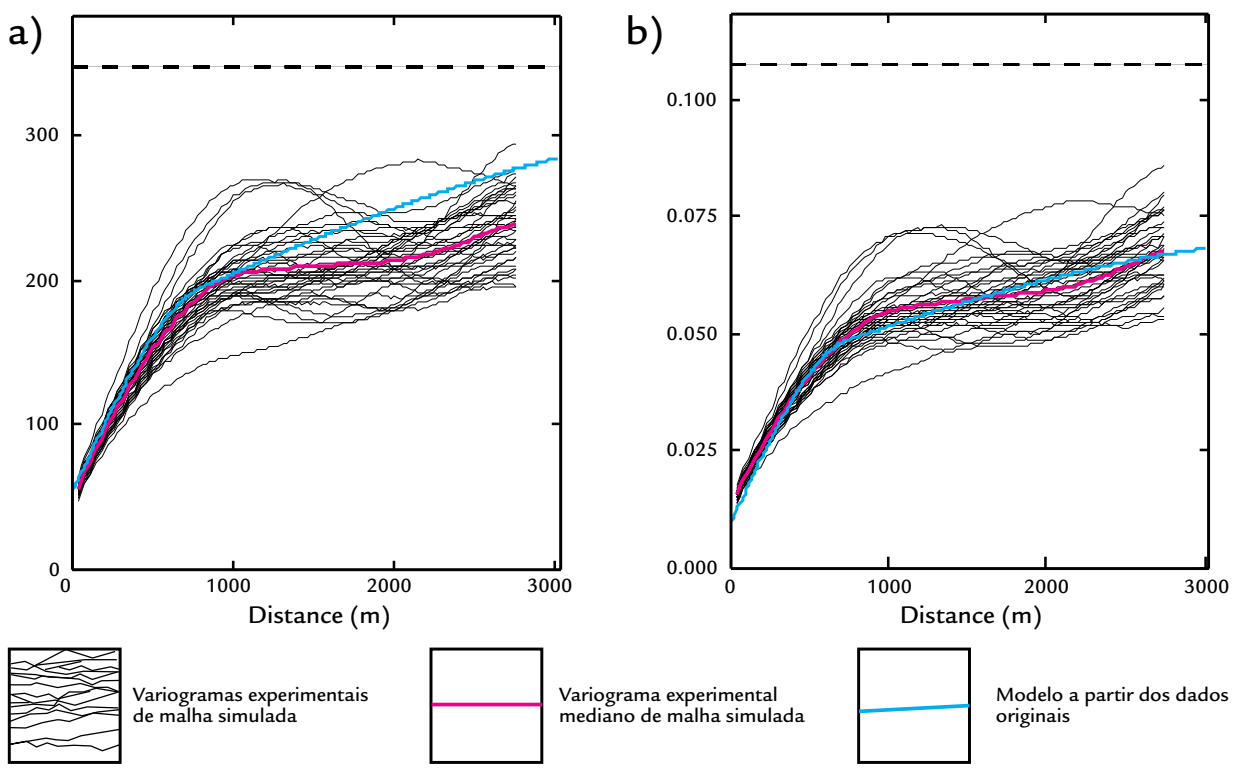

Variograma experimental mediano de malha simulada

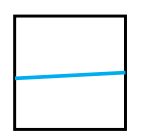

Modelo a partir dos dados originais

acumulado e da espessura, e os modelos variográficos dos dados iniciais (Figura 3).

Os equipamentos de lavra não são capazes de selecionar, precisamente, o material a ser extraído, de forma que o carvão e o estéril são lavrados com um certo grau de mistura (diluição), aumentado pela movimentação causada pelo desmonte. Para compor os teores das pilhas, foram considerados os efeitos da diluição em cada camada, somando as mesmas $10 \mathrm{~cm}$ das camadas estéreis com

\section{Cinza ROM}

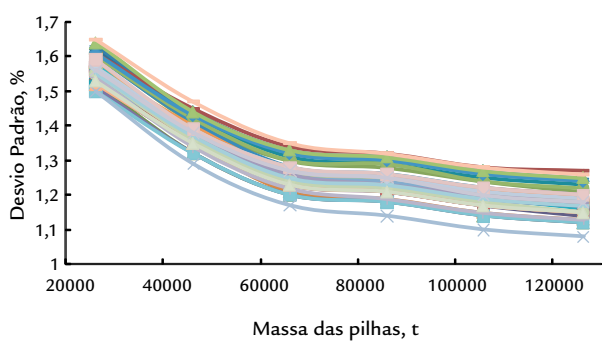

suas densidades e teores.

Para investigação do tamanho de pilha e de sua variabilidade, é necessário considerar que o tamanho da pilha seja operacional e economicamente viável. Portanto as massas de pilhas verificadas estavam compreendidas entre 30.000 a $130.000 \mathrm{t}$, aproximadamente, com intervalos de 20.000 toneladas. Com o aumento do tamanho da pilha, há redução no número de pilhas formadas ao longo da vida útil da mina e, portanto, há redução

\section{Enxofre ROM}

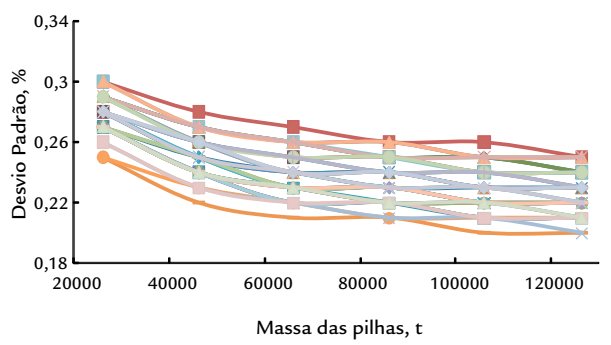

\begin{tabular}{c|c|c|c|c|c|c|c}
\hline \multicolumn{2}{c|}{} & \multicolumn{7}{c}{ Massa da pilha, kt } \\
\cline { 3 - 8 } \multicolumn{2}{c|}{} & $\mathbf{3 0}$ & $\mathbf{5 0}$ & $\mathbf{7 0}$ & $\mathbf{9 0}$ & $\mathbf{1 1 0}$ & $\mathbf{1 3 0}$ \\
\hline \multirow{3}{*}{\begin{tabular}{c}
$\begin{array}{c}\text { Conteúdos de } \\
\text { cinza (ROM), } \\
\%\end{array}$ \\
\cline { 2 - 8 }
\end{tabular}} & Limite máximo & 32.17 & 61.12 & 60.28 & 60.04 & 59.58 & 59.65 \\
\cline { 2 - 8 } & Limite mínimo & 47.37 & 47.91 & 49.24 & 50.27 & 50.70 & 50.38 \\
\cline { 2 - 8 } & Banda de incerteza & 14.8 & 13.21 & 11.04 & 9.77 & 8.88 & 8.27 \\
\hline & Redução da banda & - & $11 \%$ & $25 \%$ & $34 \%$ & $40 \%$ & $44 \%$ \\
\hline \multirow{3}{*}{$\begin{array}{c}\text { Teores de } \\
\text { enxofre (ROM), } \\
\%\end{array}$} & Limite máximo & 2.89 & 2.79 & 2.71 & 2.62 & 2.58 & 2.59 \\
\cline { 2 - 8 } & Limite mínimo & 0.67 & 0.75 & 0.85 & 0.84 & 0.88 & 0.87 \\
\cline { 2 - 8 } & Banda de incerteza & 2.22 & 2.04 & 1.86 & 1.78 & 1.70 & 1.72 \\
\cline { 2 - 8 } & Redução da banda & - & $8 \%$ & $16 \%$ & $20 \%$ & $23 \%$ & $23 \%$ \\
\hline
\end{tabular}

As simulações, também, foram validadas pela reprodução dos variogramas. Os variogramas dos atributos cinzas e enxofre diretos não puderam ser comparados devido às diferenças dos suportes amostrais. Portanto foram sobrepostos os variogramas direcionais, provenientes dos pontos simulados do conteúdo de cinzas

Figura 3

Validação dos variogramas para a variável acumulada do conteúdo de cinzas (a) e espessura (b) da camada superior na direção N-S.

dos valores médios extremos, devido à relação volume-variância (Parker, 1979), que promove homogeneização.

Para cada tamanho de pilha, há 40 sequências equiprováveis de teores, ao longo da vida útil da mina. Utilizando os teores de cada sequência, obtêm-se as médias e desvios-padrão de cada sequência. Com o aumento da massa homogeneizada, menos pilhas serão formadas e menor será o desvio-padrão entre os teores das pilhas, que formam uma sequencia, chegando ao

Figura 4

Redução do desvio-padrão dos teores entre pilhas conforme o aumento da massa homogeneizada na pilha.

Tabela 1

Limites de teores dos atributos e o intervalo da banda de confiabilidade para cada tamanho de pilha. 
Conteúdo de cinzas, \%

Figura 5

Sequência de teores máximos e mínimos dos atributos considerando a diluição, para pilhas de $70 \mathrm{kt}$. A linha escura refere-se ao nível máximo estabelecido pelo minerador para o parâmetro.

limite de toda a jazida ser representada por uma única pilha com desvio-padrão entre pilhas igual a zero e teor igual ao teor médio da jazida (situação hipotética teórica, ignorando o chamado Erro Fundamental de Heterogeneidade - Gy, 1982). Porém limites operacionais e, mais restritamente, financeiros devem impedir a escolha de massas homogeneizadas muito grandes, existindo um ponto ótimo de massa dos lotes a serem entregues ao consumidor. A Figura 4 mostra que, a partir da massa homogeneizada de 70 kt, o desvio-padrão entre pilhas decresce de forma mais suave, onde grandes acréscimos de massa não acarretariam significantes reduções de variabilidade entre lotes. Sendo assim, os resultados escolhidos, para ilustrar o comportamento dos teores, são referentes à massa de 70 mil toneladas.

A Figura 5 mostra as sequências de pilhas com seus respectivos valores dos atributos enxofre e cinza formadas para massa de 70 mil toneladas durante todo o tempo previsto de atividade da lavra. Os gráficos foram construídos a partir dos valores extremos de cada pilha ge-

Figura 6

Mapas de localização dos pontos médios das pilhas de $70 \mathrm{kt}$, com as respectivas probabilidades de excederem os limites do contrato. a pilha de $30 \mathrm{kt}$.

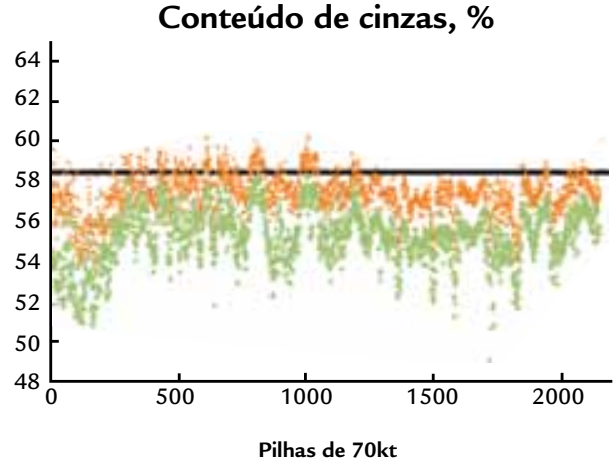

rados pelas 40 realizações. Desta forma, as sequências de pontos apresentadas não representam valores provenientes de alguma simulação específica, mas, sim, a banda de confiabilidade formada pelas simulações, incluindo o valor máximo e mínimo esperado para cada pilha em particular. Para representar o comportamento para os outros tamanhos de pilhas, a Tabela 1 mostra o porcentual de pilhas que ultrapassam os limites aceitos pelo consumidor e as reduções das bandas de incerteza relativas à incerteza medida para

Como cada bloco simulado (e, consequentemente, as pilhas formadas por esses blocos) tem uma distribuição de valores equiprováveis, há uma probabilidade de o valor do lote ultrapassar os limites estabelecidos. Assim, previsões podem ser feitas e decisões podem ser tomadas com antecedência, para adequar os lotes que apresentarem probabilidades elevadas.

Entre os 40 valores equiprováveis de cada pilha, foi feita uma contagem daqueles que ultrapassam os limites máximos estabelecidos. Desta forma, a

Probabilidade de exceder $58,5 \%$ de cinza
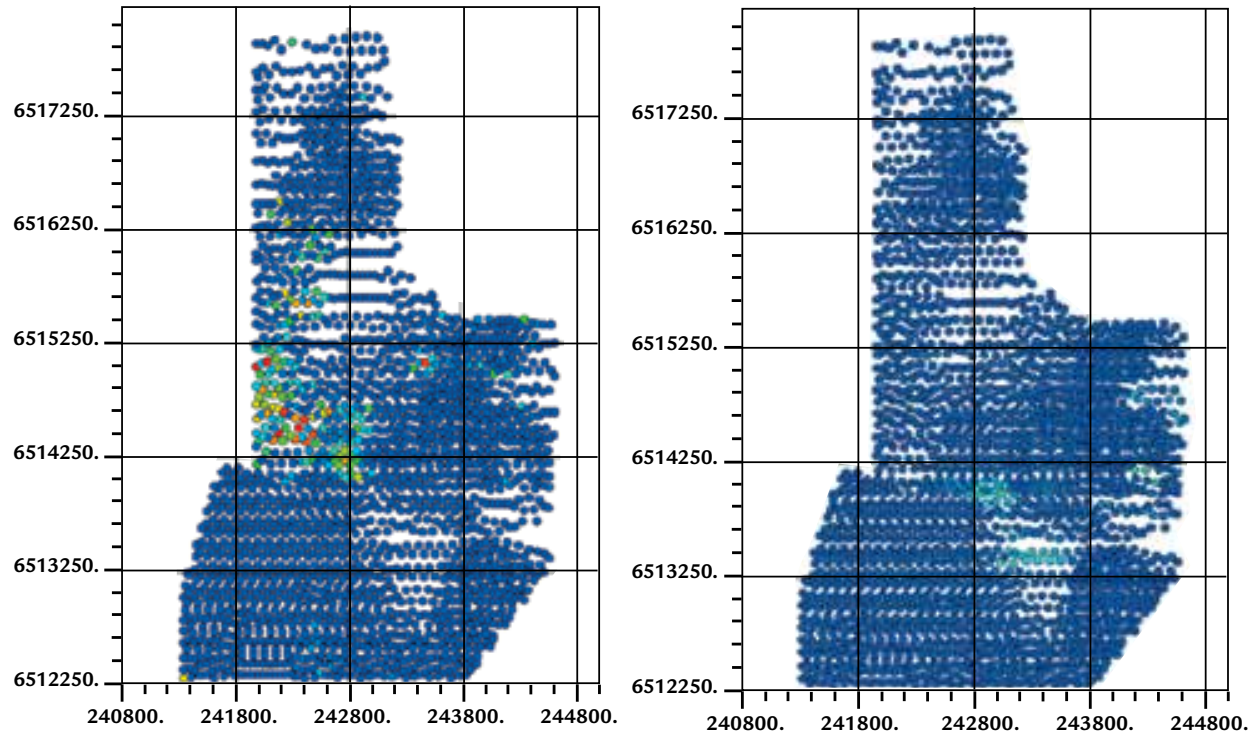

Teor de enxofre, \%

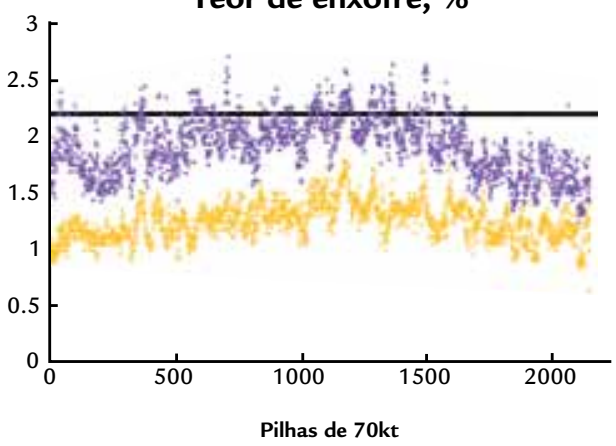

parcela de lotes calculados que estão fora dos padrões pode ser usada para se obter a probabilidade de a pilha ser passível de penalidade.

Para ilustrar os locais de maior probabilidade de valores excessivos, foram construídos mapas de localização das pilhas, para massa de 70 mil toneladas, com legenda de cores para se identificarem os locais de maior probabilidade. A Figura 6 mostra essas probabilidades calculadas para conteúdo de cinza e teor de enxofre e sua localização no depósito.

A maior parte das pilhas de $70 \mathrm{mil}$ toneladas apresenta probabilidade zero de exceder os valores aceitáveis pelo consumidor. A análise feita para o conteúdo de cinzas mostra algumas pilhas com grandes probabilidades de ultrapassarem $58,5 \%$, diferente dos resultados para teor de enxofre, onde poucos lotes apresentam probabilidade maior que zero. Porém o adensamento amostral poderia reduzir, consideravelmente, a faixa de valores resultante das simulações, fazendo com que alguns lotes tenham suas probabilidades de exceder os limites reduzidas.

Probabilidade de exceder $2,2 \%$ de enxofre

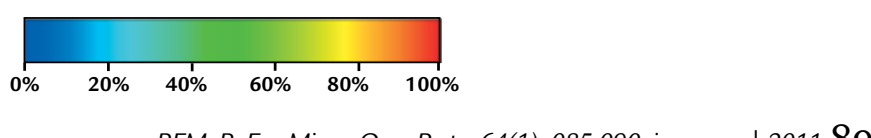




\section{Conclusões}

Usando técnicas de homogeneização por pilhas, mesmo com o custo de investimento relativamente baixo, para que se possa prever qual a variabilidade dos lotes entregues ao mercado, é indispensável o conhecimento detalhado da variabilidade do material lavrado. Nesse trabalho, técnicas de simulação geoestatística foram consideradas, pois são capazes de prever faixas de variabilidade dos teores esperados para pilhas/lotes.

Foram gerados cenários equiprováveis para conteúdo de cinza e teores de enxofre em um depósito de carvão na região Sul do país. Os modelos foram validados com procedimentos adequados. A grande vantagem da realização de vários cenários é incorporar a incerteza ao valor de lote fornecido às plantas de beneficiamento ou termelétricas.

Conforme a metodologia adotada, foi possível reduzir, consideravelmente, $\mathrm{o}$ desvio-padrão entre pilhas, apresentando reduções maiores para o conteúdo de cinzas. A redução da flutuação de teores é indispensável para garantir o bom funcionamento da usina. Nesse caso, foram calculadas as probabilidades de cada pilha ultrapassar os valores preestabelecidos.

O usuário deve escolher o tamanho de pilha com a maior redução de variabilidade dentro dos custos viáveis para sua construção, pois a metodologia confirmou a redução da variabilidade com o aumento da massa homogeneizada.

Recomenda-se um adensamento amostral da área, para que o modelo de blocos gerado seja mais consistente.

\section{Agradecimentos}

Ao Conselho Nacional de Desenvolvimento Científico e Tecnológico (CNPq), pelo auxílio financeiro. À Copelmi Mineração, representada pelos Engenheiros
Alexandre Grigorieff e José Adolfo de Carvalho Júnior, pelas valiosas informações.

\section{Referências bibliográficas}

COSTA, J. F. C. L. et al. Aperfeiçoamento da estratégia de homogeneização em pilhas chevron utilizando simulação geoestatística. REM - Revista Escola de Minas, v. 61, n. 3, p.291296, 2008.

DAVID, M. Developments in geomathematics 2: geostatistical ore reserve estimation. Amsterdam: Elsevier Scientific Publishing Company, 1977. 2v.

GAMBIN, F., COSTA, J. F. C. L., KOPPE, J.C. Estratégia de controle de qualidade de minérios na lavra utilizando simulação geoestatística. REM - Revista Escola de Minas, v. 58, n. 3, p. 193-200, 2005.

GY, P. M. Sampling of Particulate Materials: Theory and Practice (Development in Geomathematics). (2nd.) Elsevier Science Ltd., 1982. 431p. (Revised edition edition).

ISAAKS, E. H., SRIVASTAVA, R. M. Applied Geostatistics. New York: Oxford University Press, $1989.551 \mathrm{p}$.

JOURNEL, A. G. Geostatistics for conditional simulation of ore bodies. Economic Geology, 69, 1974, p.673-687.

PARKER, H. The volume variance relationship: a useful tool for mine planning. Engineering and Mining Journal, 180, p.106-123, 1979.

Artigo recebido em 11 de novembro de 2009. Aprovado em 27 de janeiro de 2010. 